Journal for ImmunoTherapy of Cancer

\section{Immune-checkpoint inhibitors plus chemotherapy versus chemotherapy as first-line treatment for patients with extensive-stage small cell lung cancer}

\author{
Fei Zhou, ${ }^{1}$ Wencheng Zhao, ${ }^{1}$ Xiaomei Gong, ${ }^{2}$ Shengxiang Ren, ${ }^{1}$ Chunxia Su, ${ }^{1}$ \\ Tao Jiang, ${ }^{3}$ Caicun Zhou ${ }^{1}$
}

To cite: Zhou F, Zhao W, Gong X, et al. Immune-checkpoint inhibitors plus chemotherapy versus chemotherapy as firstline treatment for patients with extensive-stage small cell lung cancer. Journal for ImmunoTherapy of Cancer 2020;8:e01300. doi:10.1136/ jitc-2020-001300

$F Z$, WZ and $X G$ contributed equally.

Accepted 14 August 2020

\section{Check for updates}

(c) Author(s) (or their employer(s)) 2020. Re-use permitted under CC BY-NC. No commercial re-use. See rights and permissions. Published by BMJ.

${ }^{1}$ Department of Medical Oncology, Shanghai Pulmonary Hospital, Thoracic Cancer Institute, Tongji University School of Medicine, Shanghai, China ${ }^{2}$ Department of Radiation Oncology, Shanghai Pulmonary Hospital, Thoracic Cancer Institute, Tongji University Schoo of Medicine, Shanghai, China ${ }^{3}$ Department of Pulmonary Medicine, Shanghai Respiratory Research Institute, Zhongshan Hospital, Fudan University, Shanghai, China

\section{Correspondence to}

Caicun Zhou;

caicunzhou_dr@163.com

Tao Jiang;

tonyjiangdr@163.com

\section{ABSTRACT}

We performed a meta-analysis to comprehensively investigate the efficacy and safety of immune-checkpoint inhibitors (ICls) plus chemotherapy in patients with extensive-stage small cell lung cancer (ES-SCLC). The primary outcome was overall survival (OS). The secondary outcomes included progression-free survival (PFS), objective response rate (ORR) and $\geq$ grade 3 adverse events (AEs). A total of six studies involving 2905 patients were identified, including 469 patients receiving program death ligand 1 (PD-L1) inhibitor plus chemotherapy, 308 receiving PD-1 inhibitors plus chemotherapy, 563 receiving CTLA-4 inhibitors plus chemotherapy, 268 receiving PD-L1/CTLA-4 inhibitors plus chemotherapy, and 1297 receiving chemotherapy alone. $10.8 \%(283 / 2615)$ patients had baseline brain metastases (BMs). Notably, ICls plus chemotherapy was associated with significantly improved OS (HR, 0.82; 95\% Cl, 0.75 to 0.89). Subgroup analyses revealed that PD-1 inhibitors ( $\mathrm{HR}, 0.77 ; 95 \% \mathrm{Cl}, 0.64$ to 0.92 ) and PD-L1 inhibitors (HR, $0.73 ; 95 \% \mathrm{Cl}, 0.63$ to $0.85)$ plus chemotherapy yielded a statistically significant improvement in OS while CTLA-4 inhibitors did not (HR 0.92; $95 \% \mathrm{Cl}, 0.81$ to 1.06). In patients with baseline BMs, ICls plus chemotherapy showed no survival benefits over chemotherapy alone (HR, 1.23; $95 \% \mathrm{Cl}, 0.92$ to 1.64). ICls plus chemotherapy also significantly prolonged PFS (HR, $0.81 ; 95 \% \mathrm{Cl}, 0.75$ to 0.87 ) while the pooled ORRs were comparable between ICls plus chemotherapy and chemotherapy alone (RR, 1.04; $95 \% \mathrm{Cl}, 0.99$ to 1.10). Patients treated with CTLA-4 inhibitors (relative risk (RR), $1.12 ; 95 \% \mathrm{Cl}, 0.99$ to 1.28 ) experienced more $\geq$ grade 3 AEs than those treated with PD-1/PD-L1 inhibitors (RR, 1.03; $95 \% \mathrm{Cl}, 0.96$ to 1.11). The addition of PD-1/ PD-L1 inhibitors to chemotherapy resulted in significant improvements in both PFS and OS for patients with treatment-naïve ES-SCLC, not at the cost of increased AEs.

\section{INTRODUCTION}

Small cell lung cancer (SCLC) accounts for approximately $15 \%$ of all lung cancers, characterized by a highly invasive and lethal disease. Huge efforts have been made to improve the survival of this population. However, they were almost annihilated and etoposide and platinum (EP) remains standard-of-care first-line therapy for extensive-stage SCLC for the past 30 years. ${ }^{2}$

SCLC has high tumor mutational burden (TMB) ${ }^{3}$ which is associated with more tumor neoantigens and improved efficacy ofimmunecheckpoint inhibitors (ICIs). Currently, PD-1 inhibitors, including nivolumab or pembrolizumab, have been approved by Food Drug and Administration (FDA) as a third-line or later-line therapy for patients with ES-SCLC. However, the objective response rates (ORRs) were only $10 \%-19.3 \%$ as monotherapy, ${ }^{4}$ which was lower than expected response rate and may be partially attributed to the low program death ligand 1 (PD-L1) expression on tumor cells in SCLC. ${ }^{4}$ Recent success of ICIs in combination with chemotherapy in both lung adenocarcinoma and lung squamous cell carcinoma propelled this therapeutic strategy into ES-SCLC. ${ }^{6}$ Theoretically, chemotherapy, including EP, could result in immunogenic tumor cell death, increase presentation of tumor-associated antigens, promote the maturation of dendritic cells and therefore activate the cytotoxic T-cell response. $^{8}$ Encouragingly, recent two landmark trials, IMpower133 and CASPIAN, ${ }^{9} 10$ demonstrated a synergetic antitumor effect of ICIs and EP and found that the addition of PD-L1 inhibitors (atezolizumab or durvalumab) to EP could significantly improve overall survival (OS) compared with EP alone for patients with ES-SCLC. Although the absolute improvements of progressionfree survival (PFS) were moderate, a higher 12-month PFS rates were observed in patients treated with PD-L1 inhibitors plus chemotherapy (12.6\% in IMpower $133 \%$ and $18 \%$ in CASPIAN vs $5.4 \%$ and $5.0 \%$ in controls), reflecting that the PFS benefit was durable in a subset of patients. 
Nevertheless, some disparities regarding study end points have been observed. For instance, the recent KEYNOTE-604 study reported that pembrolizumab plus EP could only statistically improve PFS compared with placebo plus EP (4.5 vs 4.3 months) for patient with ES-SCLC, the significance threshold for OS differences was not met (10.8 vs 9.7 months, HR, $0.80 ; 95 \%$ CI, 0.64 to 0.98$).{ }^{11}$ Meanwhile, in another phase 2 randomized study, nivolumab plus EP significantly improved both PFS and OS compared with EP alone. ${ }^{12}$ Such disparities arose concerns about the role of different ICIs in ES-SCLC. Therefore, we performed this meta-analysis to comprehensively investigate the efficacy and safety of ICIs plus EP in patients with ES-SCLC.

\section{METHODS}

We identified eligible trials that compared ICIs plus chemotherapy against chemotherapy alone or plus placebo in first-line setting of patients with ES-SCLC from MEDLINE, EMBASE, PubMed and the Cochrane Central Register of Controlled Trials databases with the following search terms: small cell lung cancer/carcinoma, immune checkpoint inhibitor, CTLA-4, PD-1, PD-L1, ipilimumab, nivolumab, pembrolizumab, atezolizumab, durvalumab, avelumab and randomized/controlled clinical trial. The abstracts from proceedings of the American Society of Clinical Oncology, the European Society for Medical Oncology, the American Association for Cancer Research and the World Conference on Lung Cancer were also reviewed. Studies were restricted to English language published or presented before June 1, 2020.

The primary outcome was OS. The secondary outcomes included PFS, ORR and $\geq$ grade 3 adverse events (AEs). The HRs with $95 \%$ CIs for OS and PFS, and dichotomous data for ORR were extracted. Other items included the study name, first author, year of publication, study design, study phase, number of patients, study drugs, comparators, tumor assessment criteria, follow-up time. Data were extracted independently by two authors (FZ and WZ), with discrepancies resolved by consensus.

A fixed-effect or random-effect model was adopted depending on between-study heterogeneity. Publication bias was assessed by visual inspection of a funnel plot, Begg's and Egger's tests. All data were analyzed using Review Manager V. 5.3 (RevMan; Cochrane Collaboration). Statistical significance was defined as a two-sided $\mathrm{p}<0.05$.

\section{RESULTS}

In total, six studies involving 2905 patients were identified (online supplementary figure 1) ${ }^{9-15}$ The main characteristics of the included trials are presented in table 1. Two trials compared ipilimumab plus chemotherapy with chemotherapy, two trials compared PD-1 inhibitors (pembrolizumab and nivolumab) plus chemotherapy versus chemotherapy, while two trials compared PD-L1 inhibitors (atezolizumab and durvaluamb) plus chemotherapy versus chemotherapy. Additionally, the CASPIAN study was a three-arm randomized trial that investigated durvalumab with or without tremelimumab (CTLA-4 inhibitor) plus chemotherapy versus chemotherapy alone. In brief, 469 patients received PD-L1 inhibitor plus chemotherapy, 308 received PD-1 inhibitors plus chemotherapy, 563 received CTLA-4 inhibitors plus chemotherapy, 268 received PD-L1/CTLA-4 inhibitors plus chemotherapy and 1297 received chemotherapy alone. Moreover, 10.8\% (283/2615) patients had baseline brain metastases (BMs). The median follow-up time ranged from 10.5 to 25.1 months. All of the six trials provided OS, PFS, ORR and AE information. The assessment of risk of bias is presented in online supplementary figure 2 .

ICIs plus chemotherapy was associated with a statistically significant $18 \%$ reduction in the hazard for death (HR, $0.82 ; 95 \%$ CI, 0.75 to $0.89 ; \mathrm{p}<0.001$; online supplementary figure 3a). Subgroup analyses revealed that PD-1 inhibitors (HR, 0.77; 95\% CI, 0.64 to 0.92; $\mathrm{p}=0.005)$ and PD-L1 inhibitors (HR, $0.73 ; 95 \% \mathrm{CI}, 0.63$ to 0.85 ; $\mathrm{p}<0.001$ ) plus chemotherapy yielded a statistically significant improvement in OS while CTLA-4 inhibitors did not (HR, 0.92; 95\% CI, 0.81 to $1.06 ; \mathrm{p}=0.26$ ) (figure $1 \mathrm{a}$ ). There were no significant differences between PD-1 inhibitors and PD-L1 inhibitors in terms of OS (test for subgroup difference: $\mathrm{p}=0.71$ ) but patients treated with PD-1/PD-L1 inhibitors derived more OS benefits than those treated with CTLA-4 inhibitors (test for subgroup difference: $\mathrm{p}=0.02$ ). We further stratified patients according to baseline BMs. Notably, in patients with baseline BMs, ICIs plus chemotherapy showed no survival benefits over chemotherapy alone (HR, 1.23; 95\% CI, 0.92 to $1.64 ; \mathrm{p}=0.17$ ). While, in patients without baseline BMs, PD-1/PD-L1 inhibitors (HR, $0.75 ; 95 \% \mathrm{CI}, 0.64$ to $0.87 ; \mathrm{p}=0.0001$ ) rather than CTLA-4 inhibitors (HR, 1.03; 95\% CI, 0.88 to 1.20 ; $\mathrm{p}=0.71$ ) plus chemotherapy significantly prolonged OS (online supplementary figure 4).

ICIs plus chemotherapy also significantly prolonged PFS compared with chemotherapy alone (HR, 0.81; $95 \%$ CI, 0.75 to $0.87 ; \mathrm{p}<0.001$ ) (online supplementary figure $3 \mathrm{~b})$. Subgroup analyses showed that all of PD-1 inhibitors (HR, 0.72; 95\% CI, 0.61 to 0.86 ; $\mathrm{p}=0.0002$ ), PD-L1 inhibitors (HR, 0.79; 95\% CI, 0.68 to $0.91 ; \mathrm{p}=0.001$ ), CTLA-4 inhibitors plus chemotherapy (HR, 0.86; 95\% CI, 0.76 to $0.97 ; \mathrm{p}=0.01$ ) yielded a statistically significant improvement in PFS (figure 1b). There were no significant differences among PD-1 inhibitors, PD-L1 inhibitors and CTLA-4 inhibitors regarding PFS.

The pooled ORRs were comparable between ICIs plus chemotherapy and chemotherapy alone (RR, 1.04; 95\% CI, 0.99 to $1.10 ; \mathrm{p}=0.15$ ) (online supplementary figure 3c). Subgroup analyses suggested that none of PD-1 inhibitors, PD-L1 inhibitors, CTLA-4 inhibitors improved ORRs (figure 1c).

ICIs plus chemotherapy was associated with increased zgrade 3 AEs compared with chemotherapy alone (RR, $1.07 ; 95 \%$ CI, 1.01 to $1.14 ; \mathrm{p}=0.02$ ) (online supplementary 


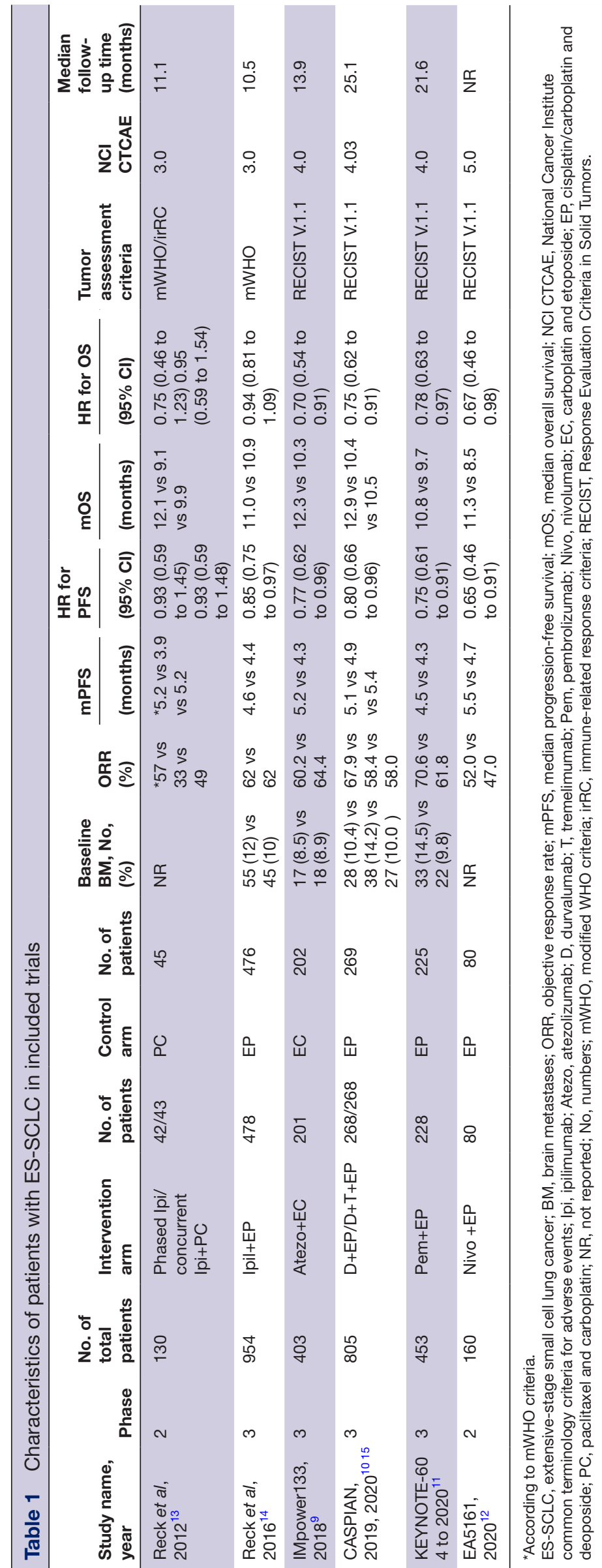


A

\section{Overall Survival}

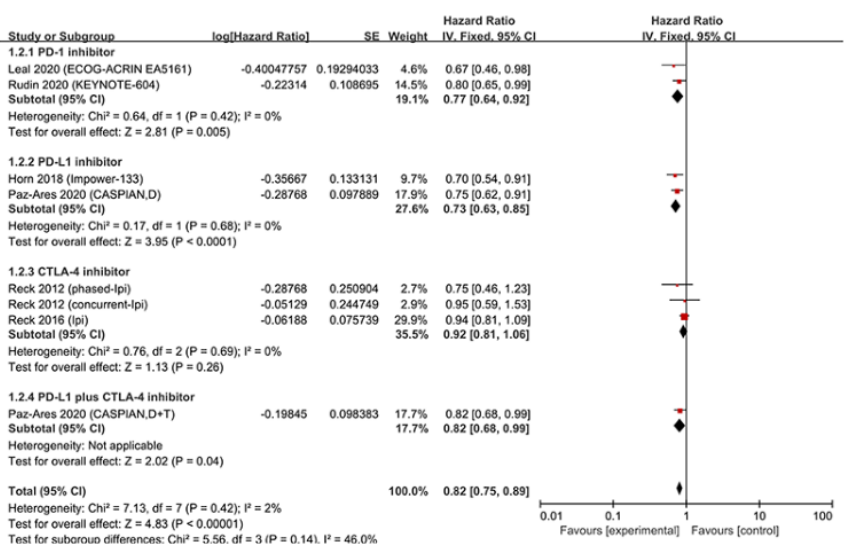

C
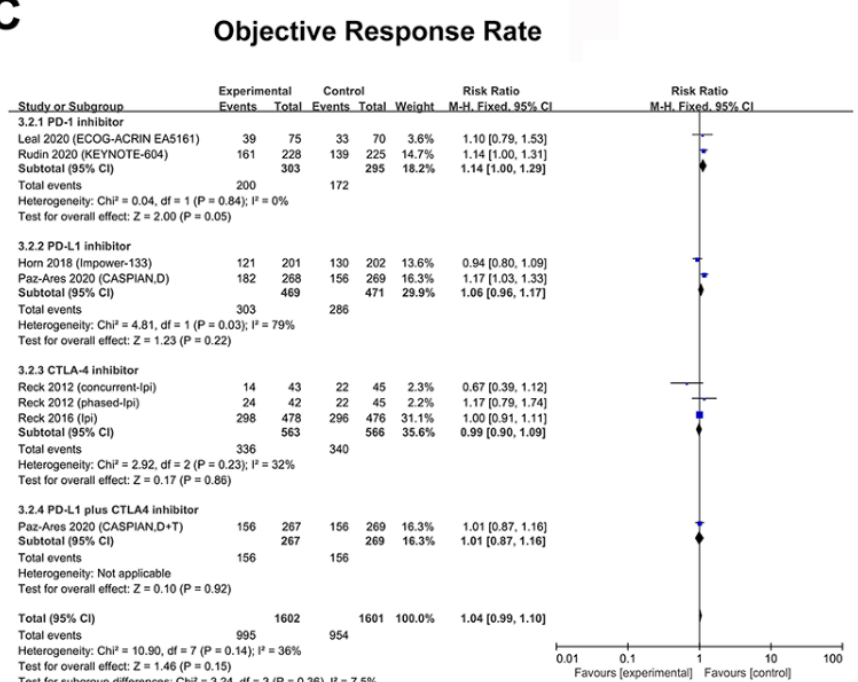

B

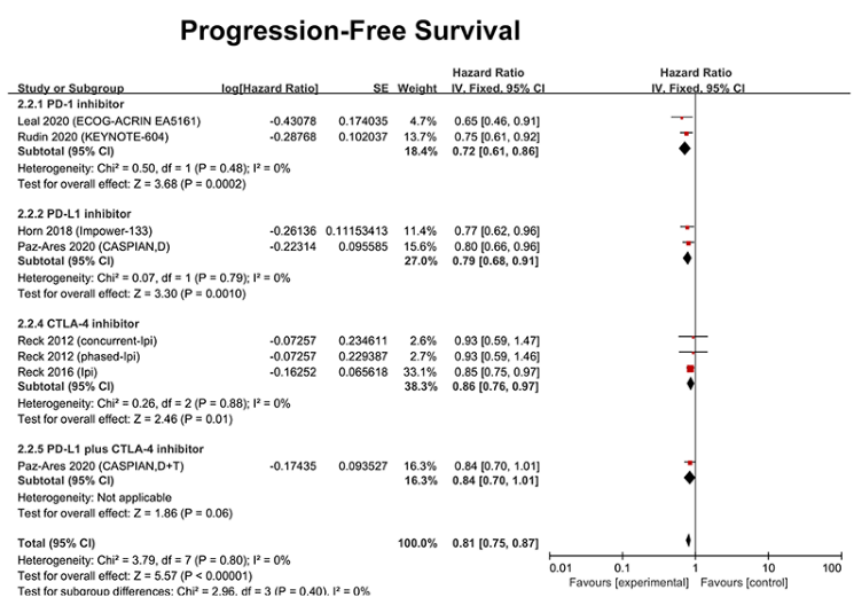

D

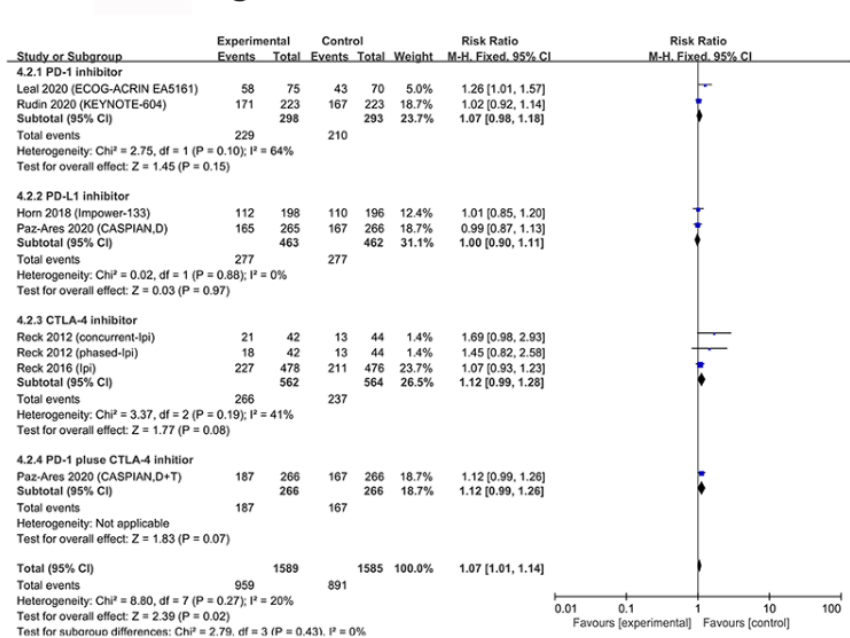

Figure 1 Subgroup analyses of immune-checkpoint inhibitors plus chemotherapy vs chemotherapy alone. (A) Overall survival, (B) progression-free survival, (C) objective response rate and (D) $\geq$ grade 3 adverse events.

figure 3d). Generally, patients treated with CTLA-4 inhibitors (RR, 1.12; 95\% CI, 0.99 to 1.28 ; $p=0.08$ ) experienced more $\geq$ grade 3 AEs than those treated with PD-1 inhibitors (RR, 1.07; 95\% CI, 0.98 to 1.18 ; $\mathrm{p}=0.15$ ) or PD-L1 inhibitors (RR, $1.00 ; 95 \% \mathrm{CI}, 0.90$ to $1.11 ; \mathrm{p}=0.97$ ), but lack of statistical significance (test for subgroup difference: $\mathrm{p}=0.25$ ) (figure $1 \mathrm{~d}$ ).

Visual inspection of the funnel plots for OS and PFS revealed no asymmetry (online supplementary figure 5), suggesting no publication bias. Begg's $(\mathrm{p}=0.536$ for OS; $p=0.536$ for PFS) and Egger's $(p=0.270$ for OS; $p=0.707$ for PFS) tests results were not significant.

\section{DISCUSSION}

To our knowledge, this was the first meta-analysis to investigate the impact of ICIs plus chemotherapy versus chemotherapy alone or plus placebo on clinical outcomes in patients with ES-SCLC. Our study demonstrated that PD-1/PD-L1 inhibitors plus chemotherapy significantly improved both PFS and OS compared with chemotherapy alone, without significantly increased $\geq$ grade 3 AEs. Despite CTLA-4 inhibitors plus chemotherapy prolonged PFS, no OS benefits were observed. In addition, the ORRs were similar between ICIs plus chemotherapy and chemotherapy alone.

The success of IMpower133 study shows the first reddening of dawn for the treatment of ES-SCLC. ${ }^{9}$ Consistent with the findings of atezolizuamb plus chemotherapy in non-squamous non-small cell lung cancer (NSCLC) (IMpower130), ${ }^{16}$ atezolizumab combined with chemotherapy also resulted in a significant improvement in terms of OS (12.3 vs 10.3 months; HR, $0.70,95 \%$ CI, 0.54 to 0.91 ) and PFS (5.2 vs 4.3 months; HR, $0.77 ; 95 \%$ CI, 0.62 to 0.96 ). The CASPIAN study also demonstrated that durvalumab plus EP significantly improved OS (13.0 vs 10.3 months; HR 0.73 ; $95 \%$ CI, 0.59 to 0.91$).{ }^{10}$ Our pooled analysis revealed a statistically significant and clinically meaningful improvement in OS for patients who received PD-L1 inhibitors plus chemotherapy (HR, 0.73; 95\% CI, 0.63 to 0.85 ), not at the cost of increased AEs 
(RR, 1.00; 95\% CI, 0.90 to 1.11), further supporting firstline use of PD-L1 inhibitors plus chemotherapy in patients with ES-SCLC. These results demonstrated the flexibility of PD-L1 inhibitors plus chemotherapy for patients with ES-SCLC. Currently, these two combinations have been approved by FDA as first-line treatment in patients with ES-SCLC.

Despite the KEYNOTE-604 study failed to meet the prespecified efficacy boundary for OS (HR, 0.80; 95\% CI, 0.64 to $0.98, \mathrm{p}=0.0164$; significance threshold $\mathrm{p}=0.0128$ ), our pooled analysis demonstrated that the addition of PD-1 inhibitors to chemotherapy also significantly improved both OS (HR, 0.77; 95\% CI, 0.64 to 0.92; $\mathrm{p}=0.005$ ) and PFS (HR, 0.72; 95\% CI, 0.61 to 0.86 ; $\mathrm{p}=0.0002$ ), suggesting PD-1 inhibitors plus chemotherapy as first-line treatment for patients with ES-SCLC deserves further investigation. In addition, our meta-analysis found that patients with baseline BMs did not derive survival benefits from PD-1/PD-L1 inhibitors plus chemotherapy. In KEYNOTE-604 study, more patients had baseline BMs than those in IMpower133 and CASPIAN studies ( $14.5 \%$ vs $8.5 \%$ vs $10 \%)$ and imbalance existed between the pembrolizumab arm and place arm regarding baseline BMs ( $14.5 \%$ vs $9.8 \%)$, which may partially explain the failure of KEYNOTE-604 study. Notably, ICIs have been demonstrated to be active and result in similar clinical outcomes in advanced NSCLC patients with BMs versus those without. ${ }^{1718}$ Therefore, the findings regarding the efficacy of ICIs in ES-SCLC patients with BMs should be interpreted with caution, as these results were based on subset analyses.

In addition, although a previous mirror meta-analysis found that PD-1 inhibitors exhibited better survival outcomes than PD-L1 inhibitors in patients with solid tumors,${ }^{19}$ our meta-analysis demonstrated no significant differences between PD-1 and PD-L1 inhibitors plus chemotherapy for patients with ES-SCLC, in terms of ORR, PFS and OS. However, the findings were obtained from indirect analysis. Nevertheless, the HRs for OS $(0.77$ vs 0.73 ) and PFS (0.72 vs 0.79 ) were comparable between the two groups, suggesting similar efficacy of PD-1 inhibitors and PD-L1 inhibitors in patients with ES-SCLC.

Our meta-analysis found no significant OS improvement in patients treated with CTLA- 4 inhibitors plus chemotherapy (HR, 0.92; 95\% CI, 0.81 to 1.06 ), which was similar with the results of ipilimumab plus chemotherapy in advanced squamous NSCLC. ${ }^{20}$ One possible explanation was that ipilimumab, which stimulates early-stage T-cell activation, may not generate an effective antitumor response in local tumor environment without corresponding effector T-cell activation. Notably, updated results from the CASPIAN study demonstrated that tremelimumab plus durvalumab and chemotherapy also failed to improve ORR, PFS and OS over chemotherapy alone. ${ }^{15}$ In contrast, AEs leading to discontinuation (21.4\% vs $10.2 \%$ vs $9.4 \%$ ) occurred more frequently in durvalumab and tremelimumab arm compared with durvalumab arm and chemotherapy-only arm. Predictive biomarkers, such as $\mathrm{TMB},{ }^{21}$ may be helpful to identify patients who may benefit from these combinations.

In conclusion, the current meta-analysis demonstrated that the addition of PD-1/PD-L1 inhibitors to chemotherapy resulted in significant improvements in both PFS and OS for patients with treatment-naïve ES-SCLC, not at the cost of increased AEs.

Contributors FZ, WZ and TJ contributed to data acquisition, data interpretation and statistical analysis and drafting of the manuscript. XG and $C Z$ contributed to the study design, data acquisition, data interpretation and statistical analysis. All the authors contributed to critical revision of the manuscript.

Funding This work was partly supported by the grants from the National Natural Science Foundation of China (No. 81703020), the Shanghai Sailing Program (No. 20YF1407500), Outstanding Young Talents Program of Shanghai Pulmonary Hospital.

Competing interests None declared.

Patient consent for publication Not required.

Provenance and peer review Not commissioned; externally peer reviewed.

Open access This is an open access article distributed in accordance with the Creative Commons Attribution Non Commercial (CC BY-NC 4.0) license, which permits others to distribute, remix, adapt, build upon this work non-commercially, and license their derivative works on different terms, provided the original work is properly cited, appropriate credit is given, any changes made indicated, and the use is non-commercial. See http://creativecommons.org/licenses/by-nc/4.0/.

\section{REFERENCES}

1 lams WT, Porter J, Horn L. Immunotherapeutic approaches for smallcell lung cancer. Nat Rev Clin Oncol 2020;17:300-12.

2 Koinis F, Kotsakis A, Georgoulias V. Small cell lung cancer (SCLC): no treatment advances in recent years. Transl Lung Cancer Res 2016;5:39-50.

3 Alexandrov LB, Nik-Zainal S, Wedge DC, et al. Signatures of mutational processes in human cancer. Nature 2013;500:415-21.

4 Antonia SJ, López-Martin JA, Bendell J, et al. Nivolumab alone and nivolumab plus ipilimumab in recurrent small-cell lung cancer (CheckMate 032): a multicentre, open-label, phase 1/2 trial. Lancet Oncol 2016;17:883-95.

5 Chung HC, Piha-Paul SA, Lopez-Martin J, et al. Pembrolizumab after two or more lines of previous therapy in patients with recurrent or metastatic SCLC: results from the KEYNOTE-028 and KEYNOTE-158 studies. J Thorac Oncol 2020;15:618-27.

6 Paz-Ares L, Luft A, Vicente D, et al. Pembrolizumab plus chemotherapy for squamous non-small-cell lung cancer. $N$ Engl J Med 2018;379:2040-51.

7 Gadgeel S, Rodríguez-Abreu D, Speranza G, et al. Updated analysis from KEYNOTE-189: pembrolizumab or placebo plus pemetrexed and platinum for previously untreated metastatic Nonsquamous nonsmall-cell lung cancer. J Clin Oncol 2020;38:1505-17.

8 Bracci L, Schiavoni G, Sistigu A, et al. Immune-based mechanisms of cytotoxic chemotherapy: implications for the design of novel and rationale-based combined treatments against cancer. Cell Death Differ 2014;21:15-25.

9 Horn L, Mansfield AS, Szczęsna A, et al. First-Line Atezolizumab plus chemotherapy in extensive-stage small-cell lung cancer. $N$ Engl $J$ Med 2018;379:2220-9.

10 Paz-Ares L, Dvorkin M, Chen Y, et al. Durvalumab plus platinumetoposide versus platinum-etoposide in first-line treatment of extensive-stage small-cell lung cancer (Caspian): a randomised, controlled, open-label, phase 3 trial. Lancet 2019;394:1929-39.

11 Rudin CM, Awad MM, Navarro A, et al. Pembrolizumab or placebo plus etoposide and platinum as first-line therapy for extensivestage small-cell lung cancer: randomized, double-blind, phase III KEYNOTE-604 study. J Clin Oncol 2020:JCO2000793.

12 Ticiana L, Yating W, Afshin D, et al. Randomized phase II clinical trial of cisplatin/carboplatin and etoposide $(\mathrm{Ce})$ alone or in combination with nivolumab as frontline therapy for extensive-stage small cell lung cancer (ES-SCLC): ECOG-ACRIN EA5161. J Clin Oncol 2020;38.

13 Reck M, Bondarenko I, Luft A, et al. Ipilimumab in combination with paclitaxel and carboplatin as first-line therapy in extensive-disease- 
small-cell lung cancer: results from a randomized, double-blind, multicenter phase 2 trial. Ann Oncol 2013;24:75-83.

14 Reck M, Luft A, Szczesna A, et al. Phase III randomized trial of ipilimumab plus etoposide and platinum versus placebo plus etoposide and platinum in extensive-stage small-cell lung cancer. $J$ Clin Oncol 2016;34:3740-8.

15 Paz-Ares LG, Dvorkin M, Chen Y, et al. Durvalumab + tremelimumab + platinum-etoposide in first-line extensive-stage SCLC (ES-SCLC): updated results from the phase III Caspian study. JCO 2020;38:9002.

16 West $\mathrm{H}$, McCleod M, Hussein M, et al. Atezolizumab in combination with carboplatin plus nab-paclitaxel chemotherapy compared with chemotherapy alone as first-line treatment for metastatic non-squamous non-small-cell lung cancer (IMpower130): a multicentre, randomised, open-label, phase 3 trial. Lancet Oncol 2019;20:924-37.

17 Goldberg SB, Schalper KA, Gettinger SN, et al. Pembrolizumab for management of patients with NSCLC and brain metastases: long- term results and biomarker analysis from a non-randomised, openlabel, phase 2 trial. Lancet Oncol 2020;21:655-63.

18 Hendriks LEL, Henon C, Auclin E, et al. Outcome of patients with non-small cell lung cancer and brain metastases treated with checkpoint inhibitors. J Thorac Oncol 2019;14:1244-54.

19 Duan J, Cui L, Zhao X, et al. Use of immunotherapy with programmed cell death 1 vs programmed cell death ligand 1 inhibitors in patients with cancer: a systematic review and metaanalysis. JAMA Oncol 2019. doi:10.1001/jamaoncol.2019.5367. [Epub ahead of print: 26 Dec 2019].

20 Govindan R, Szczesna A, Ahn M-J, et al. Phase III trial of ipilimumab combined with paclitaxel and carboplatin in advanced squamous non-small-cell lung cancer. J Clin Oncol 2017;35:3449-57.

21 Hellmann MD, Callahan MK, Awad MM, et al. Tumor mutational burden and efficacy of nivolumab monotherapy and in combination with ipilimumab in small-cell lung cancer. Cancer Cell 2018;33:853-61. 